OPEN ACCESS

Edited by:

Rey Carabeo,

Washington State University,

United States

Reviewed by:

Charles Lawrence Larson,

Rocky Mountain Laboratory,

United States

Stefania Spano,

University of Aberdeen,

United Kingdom

*Correspondence:

Li Sun

Isun@qdio.ac.ch

Received: 13 May 2017

Accepted: 24 August 2017

Published: 06 September 2017

Citation:

Sui Z-h, Xu H, Wang H, Jiang S, Chi H and Sun L (2017) Intracellular

Trafficking Pathways of Edwardsiella

tarda: From Clathrin- and

Caveolin-Mediated Endocytosis to

Endosome and Lysosome.

Front. Cell. Infect. Microbiol. 7:400 doi: $10.3389 / f c i m b .2017 .00400$

\section{Intracellular Trafficking Pathways of Edwardsiella tarda: From Clathrin- and Caveolin-Mediated Endocytosis to Endosome and Lysosome}

\author{
Zhi-hai Sui ${ }^{1,2,3}$, Haijiao Xu ${ }^{4}$, Hongda Wang ${ }^{4}$, Shuai Jiang ${ }^{1,2}$, Heng Chi ${ }^{1,2}$ and Li Sun ${ }^{1,2 *}$ \\ ${ }^{1}$ Key Laboratory of Experimental Marine Biology, Institute of Oceanology, Chinese Academy of Sciences, Qingdao, China, \\ 2 Laboratory for Marine Biology and Biotechnology, Qingdao National Laboratory for Marine Science and Technology, \\ Qingdao, China, ${ }^{3}$ University of Chinese Academy of Sciences, Beijing, China, ${ }^{4}$ State Key Laboratory of Electroanalytical \\ Chemistry, Changchun Institute of Applied Chemistry, Chinese Academy of Sciences, Changchun, China
}

Edwardsiella tarda is a Gram-negative bacterium that can infect a broad range of hosts including humans and fish. Accumulating evidences have indicated that E. tarda is able to survive and replicate in host phagocytes. However, the pathways involved in the intracellular infection of $E$. tarda are unclear. In this study, we examined the entry and endocytic trafficking of $E$. tarda in the mouse macrophage cell line RAW264.7. We found that $E$. tarda entered RAW264.7 and multiplied intracellularly in a robust manner. Cellular invasion of E. tarda was significantly impaired by inhibition of clathrin- and caveolin-mediated endocytic pathways and by inhibition of endosome acidification, but not by inhibition of macropinocytosis. Consistently, RAW264.7-infecting E. tarda was co-localized with clathrin, caveolin, and hallmarks of early and late endosomes, and intracellular E. tarda was found to exist in acid organelles. In addition, E. tarda in RAW264.7 was associated with actin and microtubule, and blocking of the functions of these cytoskeletons by inhibitors significantly decreased $E$. tarda infection. Furthermore, formaldehyde-killed $E$. tarda exhibited routes of cellular uptake and intracellular trafficking similar to that of live E. tarda. Together these results provide the first evidence that entry of live $E$. tarda into macrophages is probably a passive, virulence-independent process of phagocytosis effected by clathrin- and caveolin-mediated endocytosis and cytoskeletons, and that the intracellular traffic of $E$. tarda involves endosomes and endolysosomes.

Keywords: Edwardsiella tarda, endocytosis, endosome, lysosome, cytoskeleton

\section{INTRODUCTION}

Many serious pathogenic bacteria are capable of invading eukaryotic cells and existing as intracellular parasites (Moulder, 1985; Finlay et al., 1991; Jones and Falkow, 1996; Pieters, 2001). Cellular infection starts with bacterial adhesion to host cells, which results in internalization, either by phagocytosis or by bacteria-induced endocytosis (Finlay and Cossart, 1997). Once entrapped into the vacuoles in host cells, intracellular pathogens can take different pathways for further infection (Clemens et al., 2004). Some bacteria (e.g., Salmonella typhimurium) can thrive within the acidic vacuoles that fuse with lysosomes, while others (e.g., Mycobacterium tuberculosis) prevent 
fusion of the pathogen-containing vacuoles with lysosomes, thus maintaining a protected niche inside the host cell; still others (e.g., Listeria monocytogenes) can escape from the vacuoles and survive in the cytoplasm (Horwitz, 1988; Garcia-del Portillo and Finlay, 1995). These strategies enable the pathogens to avoid the bactericidal mechanisms of host cells and facilitate the survival and invasion of the pathogens (Gaillard et al., 1987; Clemens, 1996; Rathman et al., 1996; Ray et al., 2009; Seto et al., 2011).

Edwardsiella tarda, a Gram-negative bacterium, is a pathogen with a wide host range that includes mammals, fish, birds, and reptiles (Leung et al., 2012). E. tarda has been reported to infect humans and cause bacteremia and other medical conditions (Hirai et al., 2015). In aquaculture, E. tarda is a severe pathogen and known to affect a large number of farmed fish, resulting in heavy economic losses (Park et al., 2012). E. tarda is an intracellular pathogen with the ability to invade and replicate in host phagocytes and non-phagocytes, which is a crucial part of pathogenicity (Janda et al., 1991; Ling et al., 2000; Rao et al., 2001; Okuda et al., 2006; Ishibe et al., 2008; Leung et al., 2012; Wang et al., 2013). Recent studies showed that as a strategy of intracellular survival, E. tarda inhibits the apoptosis process of zebrafish cells but induces apoptosis and pyroptosis of mouse macrophages (Zhang et al., 2016; Zhou and Sun, 2016; Qin et al., 2017). In addition, reports have shown that once inside host cells, E. tarda could escape from the endocytic vacuoles and replicate in the cytoplasm before releasing from the cells (Strauss et al., 1997). However, the pathways involved in the process of E. tarda infection in host cells are unclear.

In this study, we aimed to gain insights into the intracellular infection process of E. tarda. For this purpose, we investigated the entry and intracellular transport of E. tarda in a mouse macrophage cell line, RAW264.7. Our results indicate a clear preference of E. tarda for certain endocytic pathways and an involvement of endosome, lysosome, and cytoskeletons in the infection process.

\section{MATERIALS AND METHODS}

\section{Reagents and Antibodies}

The inhibitors used in this study are as follows. Chlorpromazine and sucrose inhibit clathrin-mediated endocytosis; methyl- $\beta$ cyclodextrin $(\mathrm{M} \beta \mathrm{CD})$ and nystatin inhibit caveolin-mediated endocytosis; rottlerin and NSC23766 inhibit macropinocytosis; chloroquine and bafilomycin A1 inhibit acidification of endosomes; cytochalasin D and CK-636 inhibit actin polymerization; nocodazole and vinblastine depolymerize microtubles. All inhibitors were purchased from Selleck (USA) and Sigma-Aldrich (USA). All inhibitors, except sucrose, were dissolved in dimethyl sulfoxide (DMSO) (Sigma, USA) according to the manufacturer's instructions. Tubule-Tracker red kit and Lyso-Tracker red kit was purchased from Beyotime Biotechnology (Beijing, China). Fluorescein isothiocyanate (FITC), 4'-6-diamidino-2-phenylindole (DAPI), formaldehyde and paraformaldehyde (PFA) was purchased from Solarbio (Beijing, China). Latex beads $(1 \mu \mathrm{m})$ were purchased from Polysciences (USA). Mouse monoclonal antibody against clathrin heavy chain and caveolin-1, rabbit polyclonal antibodies against rab5, lamp1, and cathepsin D, phalloidin-iFluor 594 Reagent and Alexa Fluor 594-conjugated secondary antibodies were purchased from Abcam (UK) and ABclonal (USA). Rat polyclonal antibodies against $E$. tarda have been reported previously (Zhou and Sun, 2016).

\section{Cell Line}

RAW264.7, a murine monocyte-macrophage cell line, was purchased from American Tissue Culture Collection (ATCC, USA). The cells were cultured in Dulbecco's minimal Eagle's medium (DMEM) (Gibco, USA) containing 10\% fetal bovine serum (FBS) (Gibco, USA) at $37^{\circ} \mathrm{C}$ in $5 \% \mathrm{CO}_{2}$.

\section{Bacteria}

E. tarda TX1 (Zhang et al., 2008) was cultured in LuriaBertani broth (LB) medium at $28^{\circ} \mathrm{C}$. TX1 was transformed with the plasmid pGFP $_{U V}$ (purchased from Clonetech, USA), and the transformant was named TX1G, which exhibits ampicillin resistance (marker of $\mathrm{pGFP}_{\mathrm{UV}}$ ) and green fluorescence under UV light. To examine the stability of TX1G, the bacteria were subcultured continuously in LB medium without ampicillin for 7 times, and the bacteria were examined for pGFP $_{U V}$ presence and observed with a fluorescence microscope. The serum survival and $50 \%$ lethal dose $\left(\mathrm{LD}_{50}\right)$ of TX1G were determined as reported previously (Yan et al., 2012).

\section{Intracellular Replication of E. tarda}

E. tarda TX1G was grown in $\mathrm{LB}$ medium at $28^{\circ} \mathrm{C}$ to an $\mathrm{OD}_{600}$ of 0.7 . The bacteria were collected by centrifugation, washed with PBS, and resuspended in PBS. The bacteria were added to $100 \%$ confluent RAW264.7 cells in a 24-well plate at a multiplicity of infection (MOI) of 10:1, and the plate was centrifuged at $800 \mathrm{~g}$ for $10 \mathrm{~min}$, followed by incubation at $28^{\circ} \mathrm{C}$ for $2 \mathrm{~h}$. Extracellular E. tarda was killed by adding gentamicin $(100 \mu \mathrm{g} / \mathrm{ml})$ to the plate, followed by incubation at $28^{\circ} \mathrm{C}$ for $1 \mathrm{~h}$. The cells were washed three times with PBS and cultured in DMEM containing $10 \mu \mathrm{g} / \mathrm{ml}$ gentamicin for $0,2,4,6$, and $8 \mathrm{~h}$. At each time point, $500 \mu 11 \%$ Triton X-100 was added to the plate to lyse the cells, and the lysate was diluted and plated onto LB agar plates supplemented with $30 \mu \mathrm{g} / \mathrm{ml}$ tetracycline (one of the antibiotic resistance markers of TX1G). The plates were incubated at $28^{\circ} \mathrm{C}$ for $48 \mathrm{~h}$, and the colony-forming units (CFU) were counted. The colonies were verified to be TX1G by PCR. The experiment was performed in triplicate.

\section{Effects of Inhibitors on E. tarda Infection}

RAW264.7 was cultured to $100 \%$ confluence in 24 -well plates. The cells were incubated with sucrose $(300 \mathrm{mM})$, chlorpromazine $(20 \mu \mathrm{M}), \mathrm{M} \beta \mathrm{CD}(1 \mathrm{mM})$, nystatin $(100 \mu \mathrm{M})$, rottlerin $(40 \mu \mathrm{M})$, NSC23766 $(100 \mu \mathrm{M})$, chloroqiune $(40 \mu \mathrm{M})$, bafilomycin A1 $(1.6 \mu \mathrm{M})$, cytochalasin D $(2 \mu \mathrm{M}), \mathrm{CK}-636(50 \mu \mathrm{M})$, nocodazole $(2 \mu \mathrm{M})$, or vinblastine $(100 \mu \mathrm{M})$ for $1 \mathrm{~h}$. The cells were then infected with E. tarda TX1G at a MOI of 10 at $28^{\circ} \mathrm{C}$ for $2 \mathrm{~h}$. The cells were washed three times with PBS and treated with $100 \mu \mathrm{g} / \mathrm{ml}$ gentamicin for $1 \mathrm{~h}$. After treatment, the cells were washed and lysed as above. Viable bacteria in the lysate were determined by plate count as above. 


\section{Transfection of Raw264.7 with siRNAs}

All siRNAs were obtained from Ribobio (Guangzhou, China). RAW264.7 cells were cultured to about $30-40 \%$ confluence in 24 well plates. Transfection was performed with Lipofectamine ${ }^{\circledR}$ 2000 Reagent Protocol (invitrogen, USA) according to the instructions of the manufacturer. Briefly, the cells were transfected with $50 \mathrm{nM}$ siRNAs of clathrin heavy chain or caveolin-1 (Zhu et al., 2011) or a scrambled siRNA (a negative control) in Lipofectamine 2000 and Opti-MEM (Invitrogen, USA) for $6 \mathrm{~h}$. The medium was then replaced with DMEM containing $10 \% \mathrm{FBS}$, and the plate was incubated at $37^{\circ} \mathrm{C}$ with $5 \% \mathrm{CO}_{2}$ for an additional $48 \mathrm{~h}$. The expression of clathrin and caveolin-1 was determined by quantitative real time reverse transcription-PCR (qRT-PCR). The primers for PCR clathrin were Clathrin-RT-F and Clathrin-RT-R, and the primers for PCR caveolin were Caveolin-RT-F and Caveolin-RT-R (Table S1). E. tarda infection of the cells was performed as above.

\section{Fluorescence Activated Cell Sorting (FACS)}

RAW264.7 cells were treated with chlorpromazine or nystatin as above. The cells were then infected with live E. tarda TX1G as above or incubated with formaldehyde-killed E. tarda TX1G (MOI of $10: 1$ ) at $28^{\circ} \mathrm{C}$ for $2 \mathrm{~h}$. The cells were washed with PBS for three times, and extracellular fluorescence was quenched by adding $1 \mathrm{ml} 0.125 \%$ trypan blue in PBS, followed by incubation at $22^{\circ} \mathrm{C}$ for $30 \mathrm{~min}$. The cells were washed as above and suspended in $1 \mathrm{ml}$ PBS. The cells were subjected to flow cytometry analysis with a Partec CyFlow Counter (Partec GmbH, Munster, Germany), and the data were analyzed with FlowJo 7.6.1 software (TreeStar, San Carlos, CA, USA).

\section{Immunofluorescence Microscopy}

RAW264.7 was incubated with E. tarda TX1G or formaldehydefixed E. tarda TX1G in $35 \mathrm{~mm}$ confocal dishes for different time at $28^{\circ} \mathrm{C}$. The cells were fixed with $4 \%$ PFA for $20 \mathrm{~min}$ and permeabilized with $0.1 \%$ Triton-X-100 for $10 \mathrm{~min}$, followed by incubation with $5 \%$ BSA for $4 \mathrm{~h}$. The cells were stained with the rabbit or mouse primary antibodies (1:500) for overnight at $4^{\circ} \mathrm{C}$. After washing three times with PBS, the cells were incubated with secondary antibodies (Alexa Fluor 594-conjugated goat antimouse or rabbit $\operatorname{IgG})$ (1:500) for $90 \mathrm{~min}$ at room temperature. The cells were washed as above and stained with DAPI for $10 \mathrm{~min}$ at room temperature. The cells were washed as above and observed with a confocal microscope (Zeiss LSM 710). At least 100 individual bacteria were scored for co-localization in at least five random fields for each experiment. Percentages of co-localization are the average of at least three experiments.

\section{Immunofluorescence Observation of Intracellular and Extracellular E. tarda}

RAW264.7 was infected with E. tarda TX1G, washed with PBS, and fixed with PFA as above. For immunofluorescence staining of extracellular bacteria, rat anti-E. tarda antibody (1:1000) was added to the cells, and the cells were incubated at $22^{\circ} \mathrm{C}$ for $2 \mathrm{~h}$. The cells were washed as above, and goat anti-rat IgG conjugated to rhodamine red (1:1000) was added to the cells. The cells were incubated at $22^{\circ} \mathrm{C}$ for $2 \mathrm{~h}$ and washed as above. The cells were observed with a confocal microscope (Zeiss LSM 710), and intracellular and extracellular bacteria were distinguished by different colors (Drevets and Campbell, 1991; Campbell et al., 2001).

\section{Lyso-Tracker Red Labeling}

RAW264.7 were infected with E. tarda TX1G for $1.5 \mathrm{~h}$ as described above, and the cells were washed with PBS and incubated with $50 \mathrm{nM}$ Lyso-Tracker red in DMEM containing $10 \% \mathrm{FBS}$ for $30 \mathrm{~min}$ at $28^{\circ} \mathrm{C}$. The cells were washed, fixed, and stained with DAPI as above. The cells were washed with PBS and viewed with a confocal microscope as above.

\section{Labeling of Actin and Microtubules}

RAW264.7 cells were incubated with E. tarda TX1G or latex beads for $1.5 \mathrm{~h}$ as described above. The cells were fixed with $4 \%$ PFA for $20 \mathrm{~min}$ and permeabilized with $0.1 \%$ Triton-X-100 for $10 \mathrm{~min}$, followed by incubation with $5 \%$ BSA for $4 \mathrm{~h}$. The cells were stained with Phalloidin-iFluor 594 Reagent $(1: 1,000)$ or Tubule-Tracker red (1:500) for $30 \mathrm{~min}$ at room temperature. The cells were washed and stained with DAPI as above. The cells were washed with PBS and viewed with a confocal microscope as above.

\section{Statistical Analysis}

All experiments were performed at least three times, and statistical analyses were carried out with SPSS 17.0 software (SPSS Inc., Chicago, IL, USA). Data were analyzed with analysis of variance (ANOVA), and statistical significance was defined as $P<0.05$.

\section{RESULTS}

\section{Stability of GFP-Expressing E. tarda}

To facilitate the study, the GFP-expressing plasmid pGFP UV $_{\text {wa }}$ introduced into E. tarda TX1, resulting in strain TX1G. pGFP was maintained stably in TX1G after 7 subcultures without selective pressure, and the cells exhibited strong fluorescence easily detectable by microscopy (Figure S1). Compared to E. tarda TX1, TX1G exhibited similar $\mathrm{LD}_{50}\left(9.3 \times 10^{3} \mathrm{CFU} / \mathrm{g}\right)$ and serum survival rate, suggesting that the introduced $\mathrm{PGFP}$ UV had no apparent effect on the virulence property of the bacteria. As such, TX1G was used for all experimental analyses in this study.

\section{Intracellular Replication of $E$. tarda in RAW264.7}

To assess the ability of E. tarda TX1G to replicate in host cells, the bacteria were incubated with RAW264.7, and intracellular bacterial number was determined at different times. The results showed that the intracellular number of TX1G increased steadily with time from 2 to $8 \mathrm{~h}$ (Figure S2). Consistently, microscopic analysis observed entry of the bacteria into RAW264.7 and, after $2 \mathrm{~h}$ of incubation, an apparent accumulation of bacterial load inside RAW264.7 (Figure 1). As shown in Figure 1Bd, intracellular bacteria (green) were easily distinguishable from extracellular bacteria (yellow) by color. 

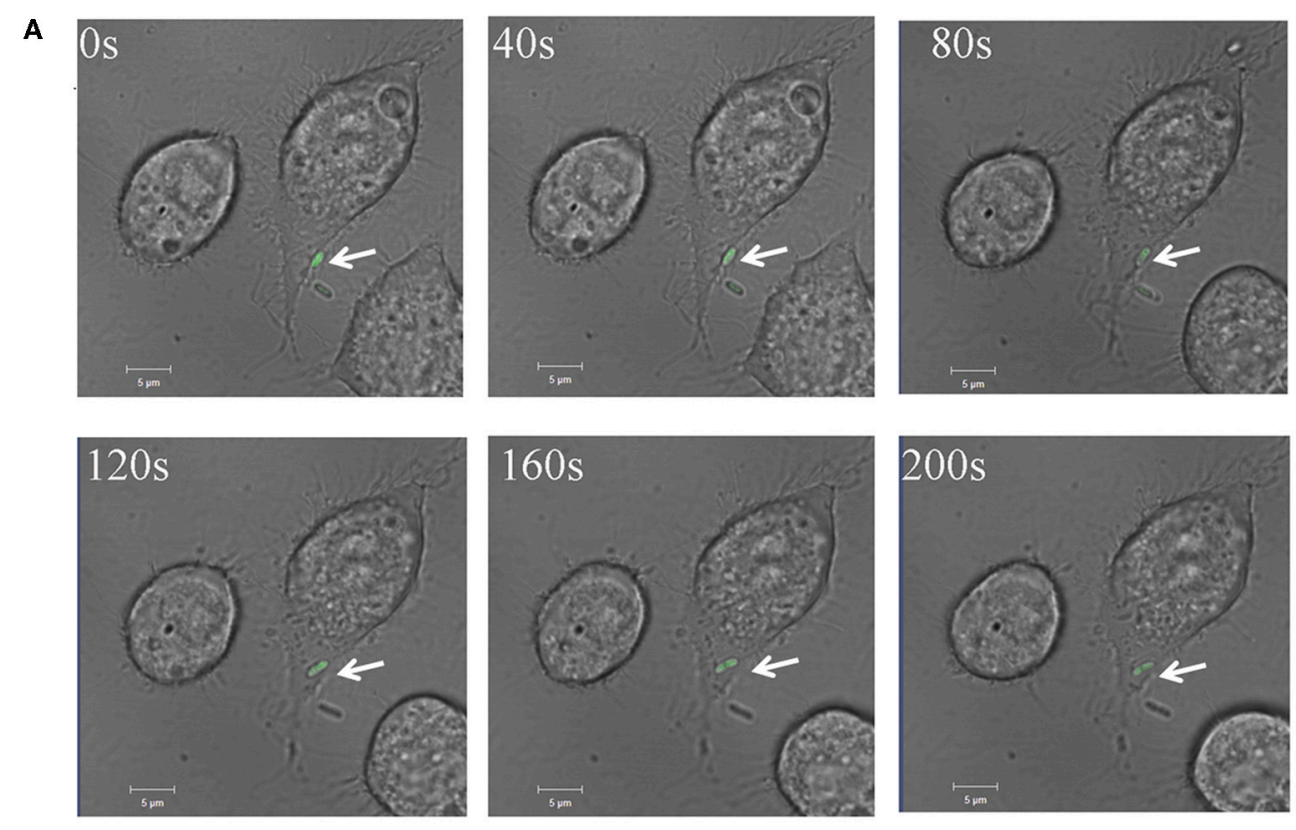

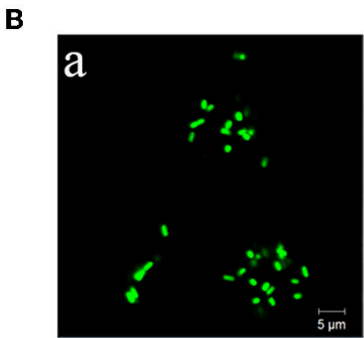

Total E. tarda

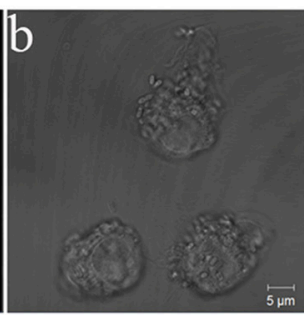

$\mathrm{BF}$

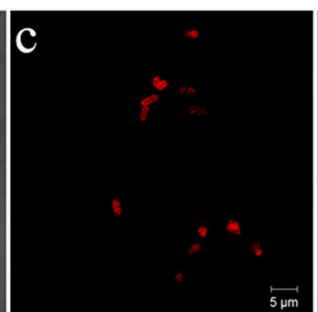

Extracellular E. tarda Merge

FIGURE 1 | Entry and replication of Edwardsiella tarda in RAW 264.7. (A) RAW264.7 was incubated with E. tarda TX1G, and infection of the bacteria into RAW264.7 was observed continuously for 200 s with a confocal microscope. Arrow indicates bacteria. (B) RAW264.7 was infected with TX1G for $2 \mathrm{~h}$ (a); the cells were then fixed, and extracellular TX1G was detected with rhodamine-labeled antibody (c). d merge of a-c.

\section{Involvement of the Endocytic Pathway in E. tarda Infection}

To examine the pathways involved in E. tarda entry into host cells, TX1G infection of RAW264.7 was conducted in the presence of inhibitors against clathrin (chlorpromazine and sucrose), caveolin (M $\beta \mathrm{CD}$ and nystatin), and macropinocytosis (NSC23766 and rottlerin). Subsequent analysis showed that in the presence of chlorpromazine, sucrose, $\mathrm{M} \beta \mathrm{CD}$, and nystatin, the relative infection ratio of TX1G decreased to $12.9,11.8$, 33.6, and 54.5, respectively, whereas the presence of NSC23766 and rottlerin had no significant effect on E. tarda infection (Figure 2A). Consistently, microscopic examination indicated that the presence of nystatin and, in particular, chlorpromazine, severely decreased the number of internalized bacteria in RAW264.7 (Figure 2B). To verify the importance of clathrinand caveolin-mediated endocytosis for E. tarda infection, the expression of clathrin and caveolin in RAW264.7 was interfered by siRNAs, which, as shown by qRT-PCR, significantly decreased the expression of clathrin and caveolin to $24.3 \pm 8.3$ and
$38.6 \pm 6.7 \%$ of that in the control cells, respectively. Subsequent infection analysis showed that in RAW264.7 with clathrin and caveolin knock-down, the uptake of E. tarda was reduced to $27.2 \pm 4.2$ and $55.7 \pm 1.9 \%$ of that in the control cells, respectively (Figure 2C). These results indicated that clathrinand caveolin-mediated pathways were vital to E. tarda infection. To examine whether these pathways were also involved in the uptake of dead bacteria, FACS analysis was performed, which showed that the presence of chlorpromazine and nystatin significantly reduced the cellular uptake of formaldehyde-killed E. tarda to $67.1 \pm 5.1$ and $73.8 \pm 3.0 \%$ of that of the control, respectively, which were roughly similar to the reduction rates $(70.2 \pm 4.5$ and $75.5 \pm 4.5 \%)$ in the uptake of live E. tarda (Figure S3).

\section{Co-Localization of Clathrin and Caveolin with Invading $E$. tarda}

Since the above results indicated a requirement of clathrinand caveolin-mediated pathways for E. tarda invasion, we 


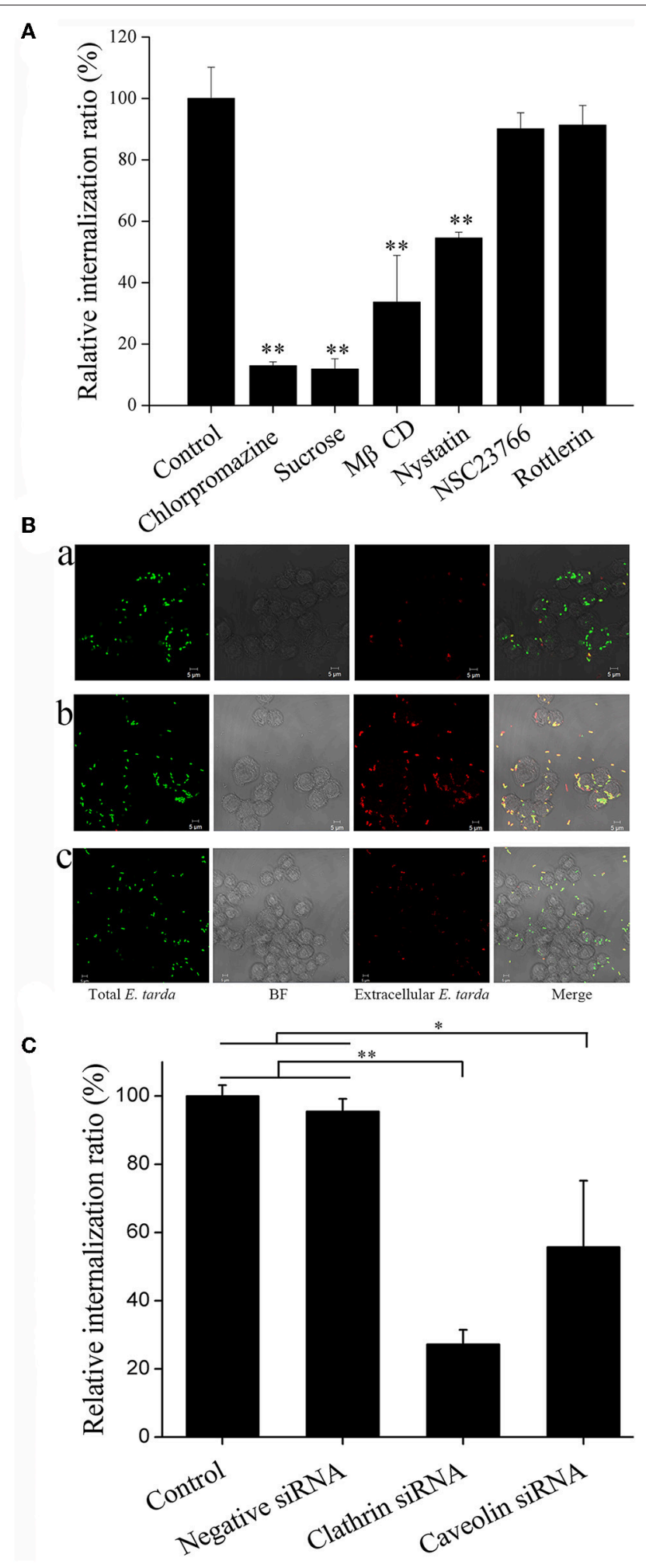

FIGURE 2 | Effect of endocytic pathway inhibitors and siRNAs on Edwardsiella tarda infection. (A) RAW264.7 was infected with E. tarda TX1G in the presence or absence (control) of various endocytic pathway inhibitors, and cellular internalization of the bacteria was determined by plate count. (B) RAW264.7 was infected with TX1G in the presence of chlorpromazine (b) and nystatin (c);
FIGURE 2 | Continued

the control cells were infected with TX1G without any inhibitor (a). The cells were fixed, and extracellular bacteria were detected with rhodamine-labeled antibody (red color). The cells were observed with a confocal microscope. Bar: $5 \mu \mathrm{m}$. (C) RAW264.7 was transfected with siRNAs of clathrin/caveolin-1 or negative control siRNAs before $E$. tarda infection, and cellular internalization of the bacteria was determined by plate count. (A) and (C), data are the means of three assays and presented as means \pm SEM. ${ }^{\star} P<0.05$, ${ }^{\star \star} P<0.01$.

examined whether E. tarda was associated with clathrin and caveolin. For this purpose, immunofluorescence microscopy was performed to examine the locations of clathrin/caveolin and E. tarda during bacterial infection of RAW264.7. The results showed that both clathrin and caveolin were colocalized with TX1G (Figure 3). The co-localization rates of bacteria with clathrin and caveolin were $23.6 \pm 6.4$ and $20.4 \pm 7.4 \%$, respectively. In addition, clathrin and caveolin distribution in uninfected control cells was also observed (Figure S4).

\section{Involvement of Endosome/Lysosome in E. tarda Infection}

To examine the potential role of endosomes in E. tarda infection, RAW264.7 was infected with TX1G in the presence of chloroquine and bafilomycin A1, inhibitors of endosome acidification. Subsequent analysis of bacterial infection showed that the relative infection ratio of TX1G decreased to $47 \pm 8$ and $46 \pm 9 \%$ of that of the control by chloroquine and bafilomycin A1, respectively. Microscopy showed that in TX1G-infected RAW264.7, the bacteria were co-localized with Rab5, a marker of early endosomes, and Lamp1 and cathepsin D, markers of late endosomes/lysosomes (Figure 4). The percentages of bacterial co-localization with Rab5, LAMP1 and cathepsin D were $29.6 \pm 6.4 \%, 28.7 \pm 8.5 \%, 36.7 \pm 7.6 \%$, respectively. Similar observation was made with formaldehyde-killed E. tarda (Figure S5). To further examine the association of lysosome with TX1G infection, TX1G-infected RAW264.7 was treated with Lysotracker red, an indicator of acid environment, and subsequent microscopy showed that co-localization of the red tracker with TX1G was detected (Figure 5).

\section{Involvement of Cytoskeletons in E. tarda Infection}

To examine whether cytoskeletons were required for E. tarda infection, RAW264.7 was infected with TX1G in the presence of actin inhibitors (cytochalasin D and CK-636) and microtubule inhibitors (nacodazole and vinblastine). Subsequent infection analysis showed that the presence of these inhibitors significantly decreased the infection ratio of TX1G to 5.3, 61.1, 48.3, and $14.1 \%$, respectively, of that of the control (Figure 6). Consistently, immunofluorescence microscopy indicated that TX1G was co-localized with phalloidin-marked actin and Tubulin-Tracker Red (Figure 7). When TX1G was replaced with latex beads, co-localization of the beads with actin and microtubule was also observed (data not shown). 

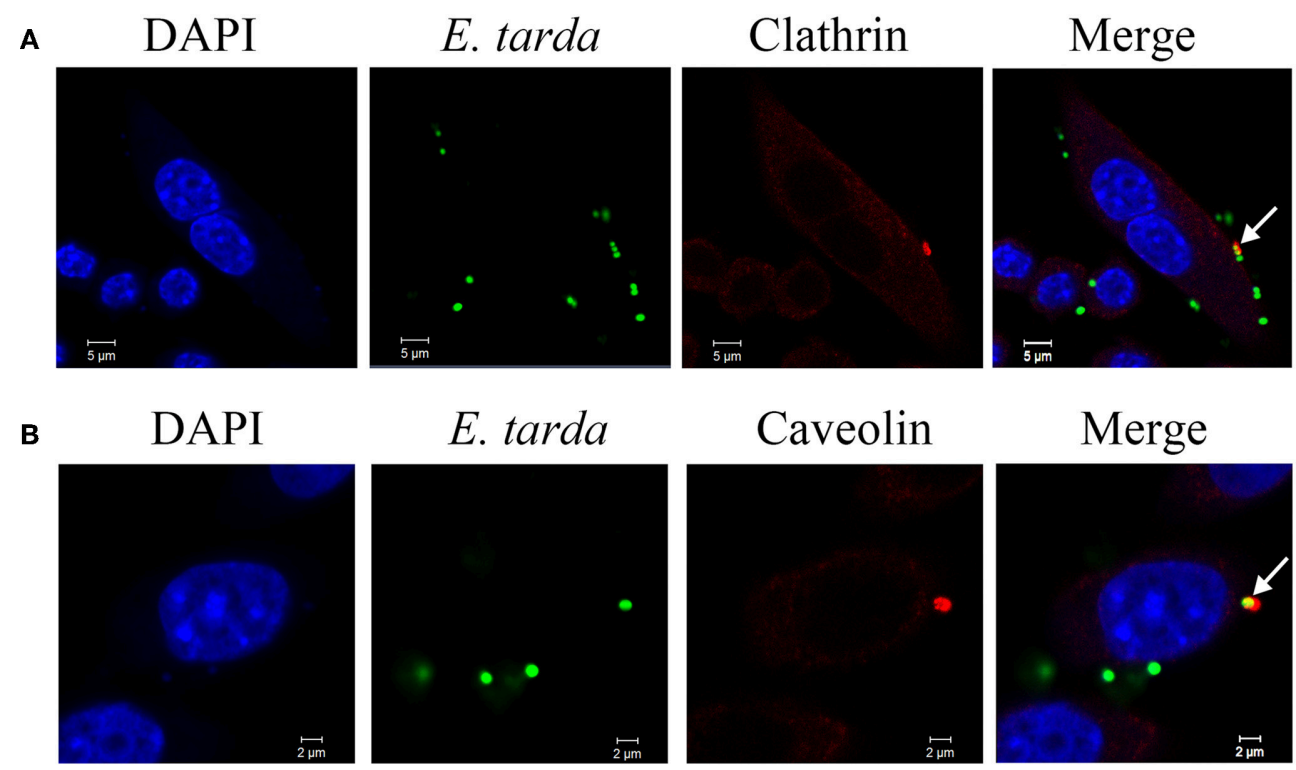

FIGURE 3 | Co-localization of Edwardsiella tarda with clathrin (A) and caveolin (B) during bacterial infection of RAW264.7. RAW264.7 cells infected with E. tarda TX1G for 30 min were treated with Alexa Fluor594-labeled antibody against clathrin or caveolin. The cells were stained with DAPI and observed with a confocal microscope. White narrows indicate co-localization of bacteria and clathrin/caveolin.

\section{DISCUSSION}

Survival in host phagocytes is an important virulence property for intracellular bacterial pathogens (Finlay and Falkow, 1997). For E. tarda, several studies have demonstrated that it is able to replicate in various types of host cells including fish and mouse macrophages (Okuda et al., 2006; Zhang et al., 2016; Qin et al., 2017). Likewise, in this study we found that E. tarda TX1G replicated steadily in RAW264.7 in a time-dependent manner, indicating an immune evasion capacity of TX1G against the bactericidal activities of macrophages. This result is consistent with our previous observation that following infection into host cells, E. tarda induces a cellular process that inhibits the initiation of apoptosis, thus enabling efficient bacterial dissemination in the host (Zhou and Sun, 2016).

Macrophages play an essential role in the immune response against pathogens (Johnston, 1988; Fujiwara and Kobayashi, 2005). Macrophages engulf microorganisms as well as other foreign particles by several mechanisms, which include phagocytosis, macropinocytosis, clathrin- and caveolinmediated endocytosis, and clathrin- and caveolin-independent endocytosis (Conner and Schmid, 2003). Pharmacological inhibitors are often used to investigate which endocytic mechanism is responsible for cellular uptake of particles (Iversen et al., 2011). In our study, we found that of the six inhibitors examined, the four inhibitors against clathrin and caveolin significantly decreased the infection of E. tarda; of these four inhibitors, chlorpromazine and sucrose targeting clathrin induced much higher levels of inhibition than $\mathrm{M} \beta \mathrm{CD}$ and nystatin targeting caveolin, suggesting that endocytosis mediated by clathrin and caveolin, especially the former, is vital to E. tarda infection. This conclusion was supported by microscopic observation, which showed co-localizations of E. tarda with clathrin and caveolin. In addition to live E. tarda, clathrin and caveolin pathways were also involved in the uptake of dead E. tarda, suggesting that these pathways were not specifically invoked by E. tarda during infection, but rather operated as common pathways during the phagocytosis process. Previous studies have shown that macropinocytosis is utilized by some pathogens, such as Salmonella, in their infection of target cells (Kerr et al., 2010). In our study, we found that unlike the inhibitors of clathrin and caveolin, the two inhibitors of macropinocytosis failed to exert any significant effect on E. tarda infection, suggesting that macropinocytosis is probably not involved in the intracellular infection of $E$. tarda.

After entry into host cells, bacterial pathogens can undergo different intracellular lifestyles, such as localizing in the cytosol or being sequestered in vesicular structures (Finlay and Cossart, 1997; Clemens et al., 2004; Bhavsar et al., 2007). For example, Legionella pneumophila entered vacuoles and inhibited phagosome acidification and maturation (Horwitz and Maxfield, 1984). In our study, we found that E. tarda was co-localized with the markers of early endosomes and late endosomes/lysosomes, and that inhibition of endosome acidification significantly decreased the infection of E. tarda. Consistently, lyso-tracker red, an indicator of acid environment, was co-localized with E. tarda. These results suggested that following internalization into RAW264.7, E. tarda was transported from early endosomes to endolysosomes, which is in agreement with the previous reports that showed E. tarda existence in the vacuoles of human epithelial cells and mouse macrophages (Fernández 

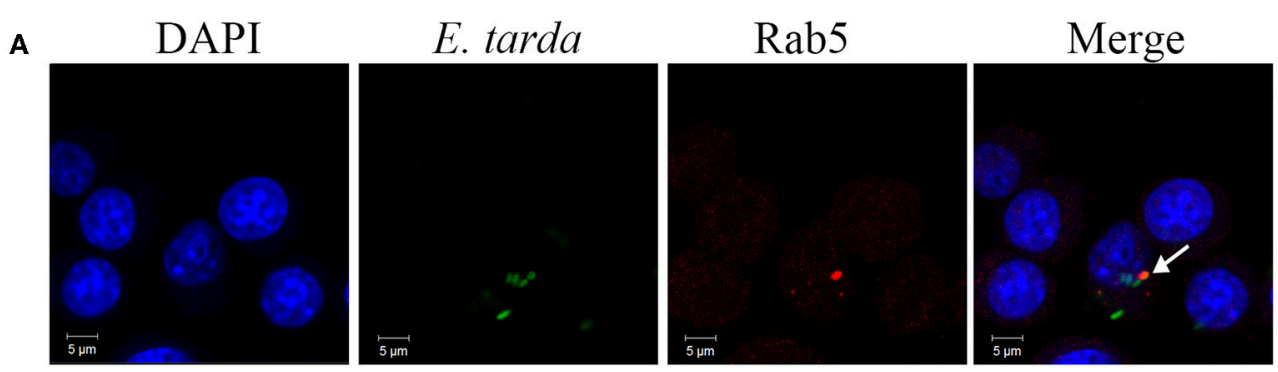

B

DAPI
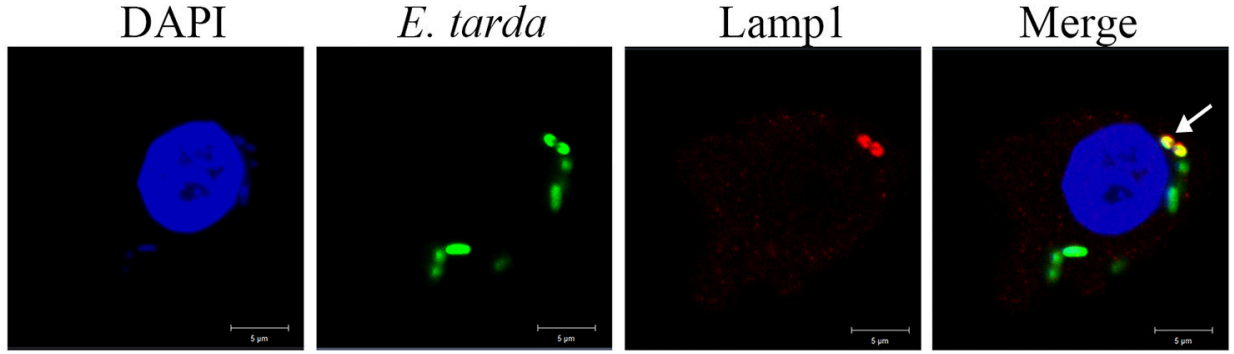

C

DAPI

E. tarda
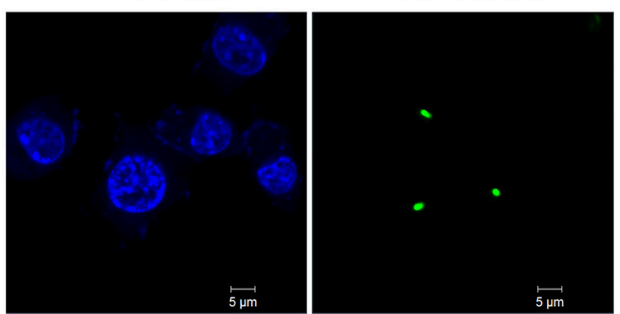

Cathepsin D

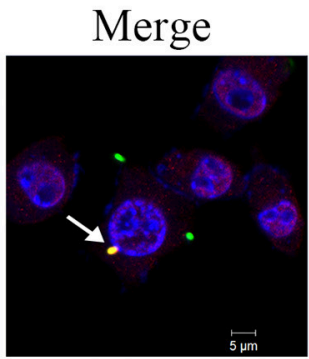

FIGURE 4 | Co-localization of Edwardsiella tarda with early and late endosome markers. RAW264.7 cells infected with E. tarda TX1G for $1 \mathrm{~h}$ (A) or $1.5 \mathrm{~h}$ (B,C) were treated with Alexa Fluor594-labeled antibody detecting Rab5 (A), Lamp1 (B), or cathepsin D (C). The cells were stained with DAPI and observed with a confocal microscope. White arrows indicate co-localization of bacteria and the respective marker.

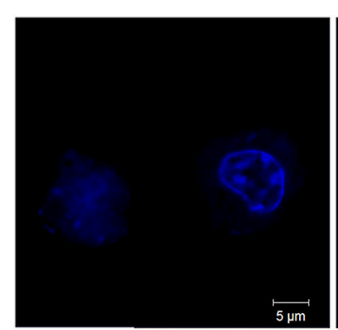

DAPI

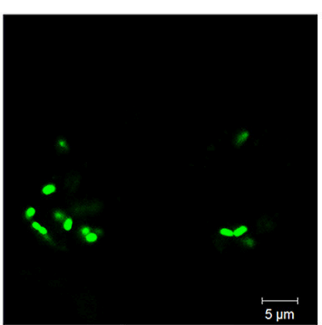

E. tarda

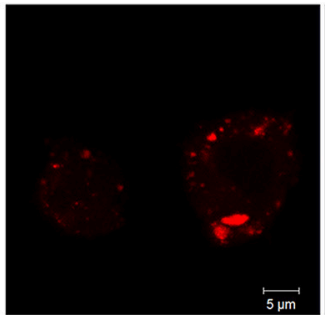

Lyso-tracker

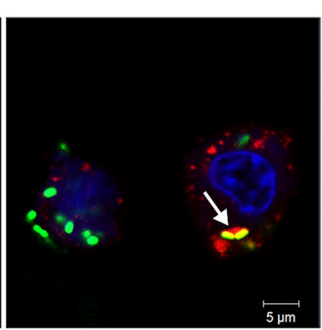

Merge

FIGURE 5 | Co-localization of Edwardsiella tarda with lysosomes in RAW264.7. RAW264.7 cells infected with E. tarda TX1G for 1.5 h were treated with Lyso-tracker red. The cells were stained with DAPI and observed with a confocalmicroscope. White arrow indicates co-localization of bacteria and lysosome.

et al., 2014; Qin et al., 2017). Since dead E. tarda was also co-localized with endosome markers, this intracellular trafficking process is very likely not involving any invasion mechanism of E. tarda. However, given the environmental stress within the endolysosomes/phagolysosomes caused by hydrolytic enzymes and low pH (Baltierra-Uribe et al., 2014), the survival of live E. tarda in these vacuoles implies possession of certain virulence mechanisms by E. tarda against the harsh conditions in the cellular compartments of host phagocytes. 

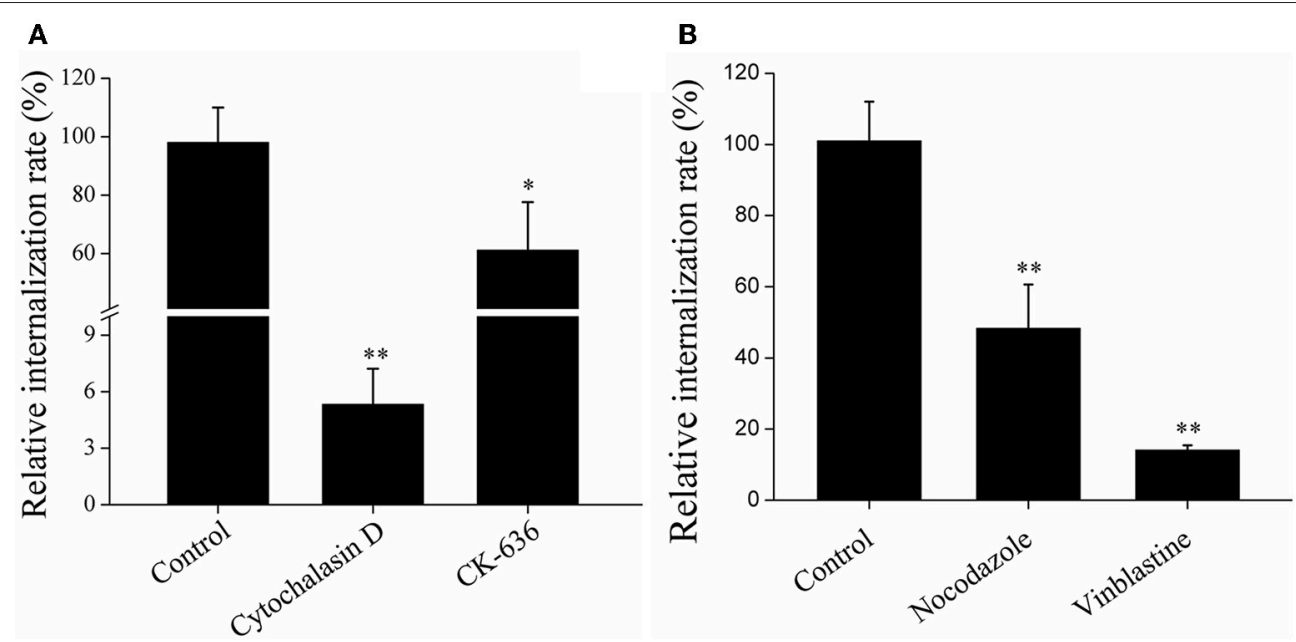

FIGURE 6 | Effects of actin (A) and microtubule (B) inhibition on Edwardsiella tarda infection. RAW264.7 was infected with E. tarda TX1G in the presence or absence (control) of the inhibitors against actin (A) or microtubule (B), and cellular internalization of the bacteria was determined by plate count. Data are the means of three independent experiments and presented as means \pm SEM. ${ }^{\star} P<0.05,{ }^{* \star} P<0.01$.

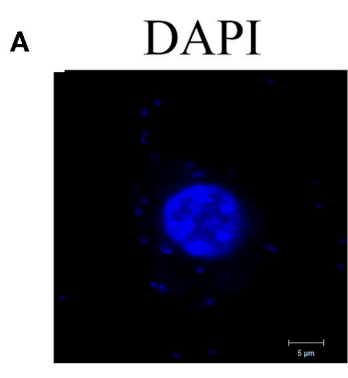

$\mathbf{B}$

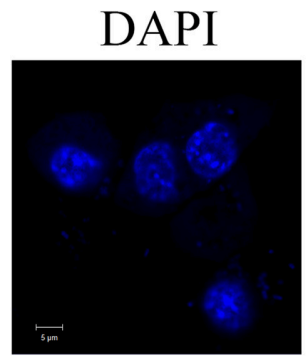

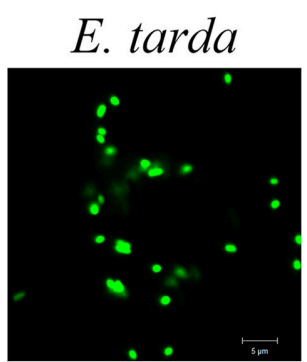

E. tarda

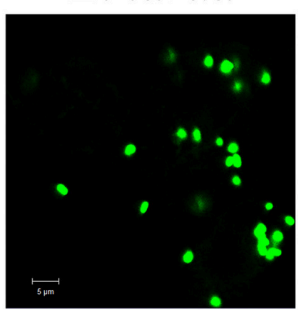

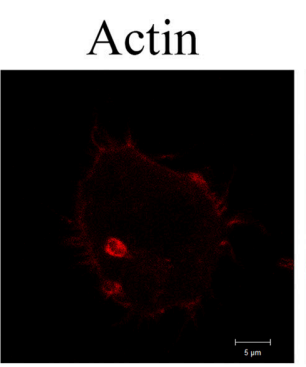

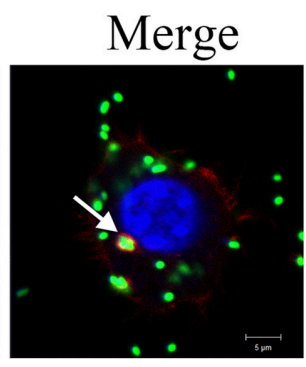

Microtubule
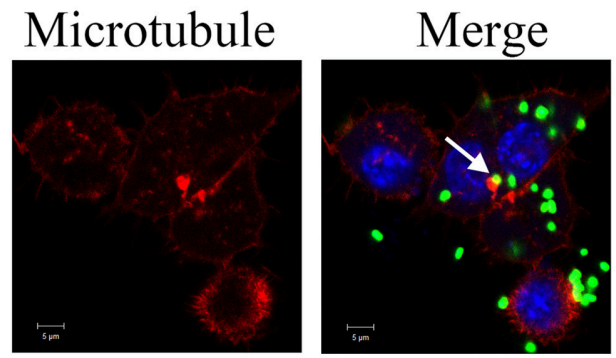

FIGURE 7 | Co-localization of actin (A) and microtubule (B) with Edwardsiella tarda in RAW264.7. RAW264.7 cells infected with E. tarda TX1G were treated with Alexa Fluor594-labeled phalloidin (A) or Tubulin-Tracker Red (B). The cells were then observed with a confocal microscope. White arrow indicates co-localization of bacteria and actin/microtubule. Bar: $5 \mu \mathrm{m}$.

Cytoskeletons provide the necessary force for the uptake of exogenous particles into membrane-bound vacuoles (Finlay and Cossart, 1997). During the process of infection, many pathogenic bacteria are model the structure of cytoskeletons to facilitate host cell infection, and cytoskeleton-dependent internalization mechanisms have been reported for several invasive organisms (Cossart and Kocks, 1994; Hu and Kopecko, 1999; Gruenheid and Finlay, 2003; Yoshida and Sasakawa, 2003; Veiga and Cossart, 2006). For example, Shigella is known to secret the VirA effector protein to destabilize microtubule to promote infection (Finlay and Cossart, 1997), and Salmonella invades host cells via a mechanism involving actin reorganization (Lim et al., 2014). In our study, we found that destruction of actin and microtubule by inhibitors significantly decreased E. tarda infection in RAW264.7, and, consistently, E. tarda was colocalized with actin and microtubule, which is in line with the previous observation that E. tarda was associated with actin in carp cells (Ling et al., 2000). These results indicated that engulfment of E. tarda depends on the normal functioning of cytoskeletons. 
In conclusion, we demonstrated for the first time that E. tarda enters, most likely in a passive manner, macrophages via the general phagocytosis pathways mediated by clathrin and caveolin, but not by macropinocytosis. Intracellular E. tarda is maintained in vacuoles, and transported from early endosomes to endolysosomes. Also, cellular infection of E. tarda requires the participation of actin and microtubule of the host cells. These results provide new insights into the intracellular invasion mechanism of E. tarda.

\section{AUTHOR CONTRIBUTION}

LS and ZS conceived and designed the experiments; ZS, SJ, and HC performed the experiments; HX and HW provided technical support for the experiments. ZS, SJ, and HC analyzed the data; ZS and LS wrote the manuscript. All authors read and approved the final manuscript.

\section{REFERENCES}

Baltierra-Uribe, S. L., Garcia-Vasquez, M. D., Castrejon-Jimenez, N. S., EstrellaPinon, M. P., Luna-Herrera, J., and Garcia-Perez, B. E. (2014). Mycobacteria entry and trafficking into endothelial cells. Can. J. Microbiol. 60, 569-577. doi: 10.1139/cjm-2014-0087

Bhavsar, A. P., Guttman, J. A., and Finlay, B. B. (2007). Manipulation of host-cell pathways by bacterial pathogens. Nature 449, 827-834. doi: 10.1038 /nature06247

Campbell, P. A., Canono, B. P., and Drevets, D. A. (2001). Measurement of bacterial ingestion and killing by macrophages. Curr. Protoc. Immunol. Chapter 14, Unit 14 116. doi: 10.1002/0471142735.im1406s 12

Clemens, D. L. (1996). Characterization of the Mycobacterium tuberculosis phagosome. Trends Microbiol. 4, 113-118. doi: 10.1016/0966-842X(96)81528-9

Clemens, D. L., Lee, B. Y., and Horwitz, M. A. (2004). Virulent and avirulent strains of Francisella tularensis prevent acidification and maturation of their phagosomes and escape into the cytoplasm in human macrophages. Infect. Immun. 72, 3204-3217. doi: 10.1128/IAI.72.6.3204-3217.2004

Conner, S. D., and Schmid, S. L. (2003). Regulated portals of entry into the cell. Nature 422, 37-44. doi: 10.1038/nature01451

Cossart, P., and Kocks, C. (1994). The actin-based motility of the facultative intracellular pathogen Listeria monocytogenes. Mol. Microbiol. 13, 395-402. doi: 10.1111/j.1365-2958.1994.tb00434.x

Drevets, D. A., and Campbell, P. A. (1991). Macrophage phagocytosis: use of fluorescence microscopy to distinguish between extracellular and intracellular bacteria. J. Immunol. Methods 142, 31-38. doi: 10.1016/0022-1759(91)90289-R

Fernández, A., Paz Villanueva, M., González, M., Fernández, F., Latif, F., Flores, S. N., et al. (2014). Adhesive and invasive capacities of Edwarsiella tarda isolated from South American sea lion. Braz. J. Microbiol. 45, 1095-1099. doi: $10.1590 /$ S1517-83822014000300044

Finlay, B. B., and Cossart, P. (1997). Exploitation of mammalian host cell functions by bacterial pathogens. Science 276, 718-725. doi: 10.1126/science.276.5313.718

Finlay, B. B., and Falkow, S. (1997). Common themes in microbial pathogenicity revisited. Microbiol. Mol. Biol. Rev. 61, 136-169.

Finlay, B. B., Ruschkowski, S., and Dedhar, S. (1991). Cytoskeletal rearrangements accompanying Salmonella entry into epithelial cells. J. Cell Sci. 99, 283-296.

Fujiwara, N., and Kobayashi, K. (2005). Macrophages in inflammation. Curr. Drug Targets Inflamm. Allergy 4, 281-286. doi: 10.2174/1568010054022024

Gaillard, J. L., Berche, P., Mounier, J., Richard, S., and Sansonetti, P. (1987). In vitro model of penetration and intracellular growth of Listeria monocytogenes in the human enterocyte-like cell line Caco-2. Infect. Immun. 55, 2822-2829.

Garcia-del Portillo, F., and Finlay, B. B. (1995). The varied lifestyles of intracellular pathogens within eukaryotic vacuolar compartments. Trends Microbiol. 3, 373-380. doi: 10.1016/S0966-842X(00)88982-9

\section{ACKNOWLEDGMENTS}

We thank Dr. Julian A. Guttman of Simon Fraser University for technical advice and useful discussion. This work was supported by the National Natural Science Foundation of China grant (31330081), the AoShan Talents Program and the Scientific and Technological Innovation Project Supported by Qingdao National Laboratory for Marine Science and Technology (No. 2015ASTP and No. 2015ASKJ02), and the Taishan Scholar Program of Shandong Province.

\section{SUPPLEMENTARY MATERIAL}

The Supplementary Material for this article can be found online at: http://journal.frontiersin.org/article/10.3389/fcimb. 2017.00400/full\#supplementary-material

Gruenheid, S., and Finlay, B. B. (2003). Microbial pathogenesis and cytoskeletal function. Nature 422, 775-781. doi: 10.1038/nature01603

Hirai, Y., Asahata-Tago, S., Ainoda, Y., Fujita, T., and Kikuchi, K. (2015). Edwardsiella tarda bacteremia. A rare but fatal water- and foodborne infection: review of the literature and clinical cases from a single centre. Can. J. Infect. Dis. Med. Microbiol. 26, 313-318. doi: 10.1155/2015/702615

Horwitz, M. A. (1988). Intracellular parasitism. Curr. Opin. Immunol. 1, 41-46. doi: 10.1016/0952-7915(88)90049-0

Horwitz, M. A., and Maxfield, F. R. (1984). Legionella pneumophila inhibits acidification of its phagosome in human monocytes. J. Cell Biol. 99, 1936-1943. doi: $10.1083 /$ jcb.99.6.1936

Hu, L., and Kopecko, D. J. (1999). Campylobacter jejuni 81-176 associates with microtubules and dynein during invasion of human intestinal cells. Infect. Immun. 67, 4171-4182.

Ishibe, K., Osatomi, K., Hara, K., Kanai, K., Yamaguchi, K., and Oda, T. (2008). Comparison of the responses of peritoneal macrophages from Japanese flounder (Paralichthys olivaceus) against high virulent and low virulent strains of Edwardsiella tarda. Fish Shellfish Immunol. 24, 243-251. doi: 10.1016/j.fsi.2007.11.001

Iversen, T. G., Skotland, T., and Sandvig, K. (2011). Endocytosis and intracellular transport of nanoparticles: present knowledge and need for future studies. Nano Today 6, 176-185. doi: 10.1016/j.nantod.2011.02.003

Janda, J. M., Abbott, S. L., and Oshiro, L. S. (1991). Penetration and replication of Edwardsiella spp. in HEp-2 cells. Infect. Immun. 59, 154-161.

Johnston, R. B. Jr. (1988). Monocytes and macrophages. New Engl. J. Med. 318, 747-752. doi: 10.1056/NEJM198803243181205

Jones, B. D., and Falkow, S. (1996). SALMONELLOSIS: host immune responses and bacterial virulence determinants ${ }^{1}$. Annu. Rev. Immunol. 14, 533-561. doi: 10.1146/annurev.immunol.14.1.533

Kerr, M. C., Wang, J. T., Castro, N. A., Hamilton, N. A., Town, L., Brown, D. L., et al. (2010). Inhibition of the PtdIns (5) kinase PIKfyve disrupts intracellular replication of Salmonella. EMBO J. 29, 1331-1347. doi: 10.1038/emboj.2 010.28

Leung, K. Y., Siame, B. A., Tenkink, B. J., Noort, R. J., and Mok, Y. K. (2012). Edwardsiella tarda virulence mechanisms of an emerging gastroenteritis pathogen. Microbes Infect. 14, 26-34. doi: 10.1016/j.micinf.2011.08.005

Lim, J. S., Shin, M., Kim, H. J., Kim, K. S., Choy, H. E., and Cho, K. A. (2014). Caveolin-1 mediates Salmonella invasion via the regulation of SopEdependent Rac1 activation and actin reorganization. J. Infect. Dis. 210, 793-802. doi: 10.1093/infdis/jiu152

Ling, S. H. M., Wang, X. H., Xie, L., Lim, T. M., and Leung, K. Y. (2000). Use of green fluorescent protein (GFP) to study the invasion pathways of Edwardsiella tarda in in vivo and in vitro fish models. Microbiology 146, 7-19. doi: 10.1099/00221287-146-1-7 
Moulder, J. W. (1985). Comparative biology of intracellular parasitism. Microbiol. Rev. 49, 298-337.

Okuda, J., Arikawa, Y., Takeuchi, Y., Mahmoud, M. M., Suzaki, E., Kataoka, K., et al. (2006). Intracellular replication of Edwardsiella tarda in murine macrophage is dependent on the type III secretion system and induces an up-regulation of anti-apoptotic NF- $\mathrm{KB}$ target genes protecting the macrophage from staurosporine-induced apoptosis. Microb. Pathog. 41, 226-240. doi: 10.1016/j.micpath.2006.08.002

Park, S. B., Aoki, T., and Jung, T. S. (2012). Pathogenesis of and strategies for preventing Edwardsiella tarda infection in fish. Vet. Res. 43:67. doi: 10.1186/1297-9716-43-67

Pieters, J. (2001). Entry and survival of pathogenic mycobacteriain macrophages. Microb. Infect. 3, 249-255. doi: 10.1016/S1286-4579(01)01376-4

Qin, L., Sun, Y. Y., Zhao, Y. J., Xu, J., and Bi, K. R. (2017). In vitro model to estimate Edwardsiella tarda-macrophage interactions using RAW264.7 cells. Fish Shellfish Immunol. 60, 177-184. doi: 10.1016/j.fsi.2016.11.027

Rao, P. S. S., Lim, T. M., and Leung, K. Y. (2001). Opsonized virulent Edwardsiella tarda strains are able to adhere to and survive and replicate within fish phagocytes but fail to stimulate reactive oxygen intermediates. Infect. Immun. 69, 5689-5697. doi: 10.1128/IAI.69.9.5689-5697.2001

Rathman, M., Sjaastad, M. D., and Falkow, S. (1996). Acidification of phagosomes containing Salmonella typhimurium in murine macrophages. Infect. Immun. 64, 2765-2773.

Ray, K., Marteyn, B., Sansonetti, P. J., and Tang, C. M. (2009). Life on the inside: the intracellular lifestyle of cytosolic bacteria. Nat. Rev. Microbiol. 7, 333-340. doi: $10.1038 /$ nrmicro2112

Seto, S., Tsujimura, K., and Koide, Y. (2011). Rab GTPases regulating phagosome maturation are differentially recruited to mycobacterial phagosomes. Traffic 12 , 407-420. doi: 10.1111/j.1600-0854.2011.01165.x

Strauss, E. J., Ghori, N., and Falkow, S. (1997). An Edwardsiella tarda strain containing a mutation in a gene with homology to shlB and $\mathrm{hpmB}$ is defective for entry into epithelial cells in culture. Infect. Immun. 65, 3924-3932.

Veiga, E., and Cossart, P. (2006). The role of clathrin-dependent endocytosis in bacterial internalization. Trends Cell Biol. 16, 499-504. doi: 10.1016/j.tcb.2006.08.005
Wang, B., Yu, T., Dong, X., Zhang, Z., Song, L., Xu, Y., et al. (2013). Edwardsiella tarda invasion of fish cell lines and the activation of divergent cell death pathways. Vet. Microbiol. 163, 282-289. doi: 10.1016/j.vetmic.2012. 12.027

Yan, M. C., Wang, X. P., Hu, W. L., Chen, S. B., Zhang, S. L., and Xie, Q. L. (2012). Visualization of Sparus macrocephalus infection by GFP-labeled Edwardsiella tarda. Isr. J. Aqua. Bamidgeh. 64, 7. Available online at: http://hdl.handle.net/ $10524 / 22920$

Yoshida, S., and Sasakawa, C. (2003). Exploiting host microtubule dynamics: a new aspect of bacterial invasion. Trends Microbiol. 11, 139-143. doi: 10.1016/S0966-842X(03)00023-4

Zhang, L., Ni, C., Xu, W., Dai, T., Yang, D., Wang, Q., et al. (2016). Intramacrophage infection reinforces the virulence of Edwardsiella tarda. J. Bacteriol. 198, 1534-1542. doi: 10.1128/JB.00978-15

Zhang, M., Sun, K., and Sun, L. (2008). Regulation of autoinducer 2 production and luxS expression in a pathogenic Edwardsiella tarda strain. Microbiology 154, 2060-2069. doi: 10.1099/mic.0.2008/017343-0

Zhou, Z. J., and Sun, L. (2016). Edwardsiella tarda-Induced inhibition of apoptosis: a strategy for intracellular survival. Front. Cell. Infect. Microbiol. 6:76. doi: 10.3389/fcimb.2016.00076

Zhu, X. D., Zhuang, Y., Ben, J. J., Qian, L. L., Huang, H. P., Bai, H., et al. (2011). Caveolae-dependent endocytosis is required for class A macrophage scavenger receptor-mediated apoptosis in macrophages. J. Biol. Chem. 286, 8231-8239. doi: $10.1074 /$ jbc.M110.145888

Conflict of Interest Statement: The authors declare that the research was conducted in the absence of any commercial or financial relationships that could be construed as a potential conflict of interest.

Copyright $\odot 2017$ Sui, Xu, Wang, Jiang, Chi and Sun. This is an open-access article distributed under the terms of the Creative Commons Attribution License (CC BY). The use, distribution or reproduction in other forums is permitted, provided the original author(s) or licensor are credited and that the original publication in this journal is cited, in accordance with accepted academic practice. No use, distribution or reproduction is permitted which does not comply with these terms. 